\title{
Performance of MALDI-TOF Mass Spectrometry to Determine the Sex of Mosquitoes and Identify Specific Colonies from French Polynesia
}

\author{
Fatou Kiné Fall, ${ }^{1,2,3}$ Maureen Laroche, ${ }^{1,2}$ Hervé Bossin, ${ }^{4}$ Didier Musso, ${ }^{1,5}$ and Philippe Parola ${ }^{1,2 *}$ \\ ${ }^{1}$ Aix Marseille Univ, IRD, AP-HM, SSA, VITROME, Marseille, France; ${ }^{2} I H U$ Méditerranée Infection, Marseille, France; ${ }^{3}$ Campus International \\ IRD-UCAD de I'IRD, Dakar, Senegal; ${ }^{4}$ Medical Entomology Laboratory, Institut Louis Malardé, Tahiti, French Polynesia; ${ }^{5}$ SELAS Eurofins \\ Labazur Guyane, Cayenne, French Guiana
}

\begin{abstract}
Mosquitoes are the main arthropod vectors of human pathogens. The current methods for mosquito identification include morphological and molecular methods. Matrix-assisted laser desorption/ionization time-of-flight mass spectrometry (MALDI-TOF MS), now routinely used for bacterial identification, has recently emerged in the field of entomology. The aim of this study was to use MALDI-TOF MS to identify mosquito colonies from French Polynesia. Five hundred specimens from French Polynesia belonging to three species, Aedes aegypti, Aedes polynesiensis, and Culex quinquefasciatus, were included in the study. Testing the legs of these mosquitoes by MALDI-TOF MS revealed a $100 \%$ correct identification of all specimens at the species level. The MALDI-TOF MS profiles obtained allowed differentiation of male from female mosquitoes and the specific identification of female mosquito colonies of the same species but different geographic origin.
\end{abstract}

\section{INTRODUCTION}

Mosquito-borne diseases are a major public health problem, particularly in tropical areas. They are responsible for more than one billion human infections and more than one million deaths worldwide per year. ${ }^{1}$ In French Polynesia, a French overseas territory located in the South Pacific (Figure 1), 16 species of mosquito have been identified, and 13 of them are known to bite humans. ${ }^{2}$ The main mosquito vectors in French Polynesia are Aedes aegypti, Aedes polynesiensis, and Culex quinquefasciatus. ${ }^{3}$ These three species are present in abundance in the five archipelagos of French Polynesia and are responsible for the transmission of not only filariae but also dengue (DENV), Zika (ZIKV), chikungunya $(\mathrm{CHIKV})$, and Ross River viruses (RRV). ${ }^{2}$

There, Ae. aegypti is the main vector of DENV, ZIKV, and $\mathrm{CHIKV},{ }^{4-7}$ whereas Ae. polynesiensis is the main vector of lymphatic filariasis and a secondary vector for $\mathrm{CHIKV}$ and Ross River virus (RRV). ${ }^{8-10}$ Culex quinquefasciatus is a potential vector for lymphatic filariasis. ${ }^{8,11}$ Native to the Pacific, Ae. polynesiensis is thought to have been widely spread in the islands of the archipelago by Polynesians a 1,000 years ago. ${ }^{12}$ Aedes aegypti and $C$. quinquefasciatus were introduced to the Pacific in the late nineteenth and early twentieth centuries. Aedes aegypti is now found in the five archipelagos of French Polynesia, including in Tahiti, the most populated island. ${ }^{9}$

Entomological surveys are essential for risk assessment and control of vector-borne diseases. These surveys include identification of mosquito vector species as an important step toward implementing vector control strategies. ${ }^{13}$ The identification of mosquitoes relies mainly on morphological and molecular biology techniques. Morphological identification, based on taxonomic keys, ${ }^{14}$ can be highly challenging in the case of damaged specimens or morphological similarities of species belonging to the same complex. ${ }^{15,16}$ It also requires entomological expertise and considerable handling time. Molecular biology, that is, PCR and sequencing, has allowed

*Address correspondence to Philippe Parola, VITROME, IHU Méditerranée Infection, 19-21 Blvd., Jean Moulin, Marseille 13005, France. E-mail: philippe.parola@univ-amu.fr significant advances by specifically identifying a large number of arthropods. ${ }^{17,18}$ However, it requires comprehensive databases (National Center for Biotechnology Information [NCBI] GenBank) and appropriate primers depending on the arthropod family and even species complex. ${ }^{19}$ There are no ideal universal primers for identifying arthropods with certainty. ${ }^{16}$ The financial cost of this technique may also limit its use, especially when it comes to identifying a large number of samples.

In recent years, a new approach has been developed for the rapid and cost-effective identification of arthropods. Matrixassisted laser desorption/ionization time-of-flight mass spectrometry (MALDI-TOF MS) is based on the comparison of the protein profiles of samples with a database of reference spectra. ${ }^{20}$ It has revolutionized the field of clinical microbiology $^{21}$ and has been successfully used and validated in several laboratory or field tests to identify arthropod vectors such as mosquitoes, ${ }^{22-24}$ fleas, ${ }^{25}$ ticks, ${ }^{26-28}$ sandflies, ${ }^{29,30}$ tsetse flies, ${ }^{31}$ and other arthropods. ${ }^{32-35}$

The aim of this study was to use MALDI-TOF MS to identify mosquitoes from French Polynesia and to test the ability of this method to distinguish sex and also geographical origin within female mosquitoes of the same species.

\section{MATERIAL AND METHODS}

Ethical considerations. The mosquito collection protocol was not submitted to an Ethics Committee for approval, as the mosquitoes used in this study were not collected from humans or animals.

Mosquitoes. Specimens of Ae. aegypti and Ae. polynesiensis used in this study include laboratory colonies established from specimens collected in French Polynesia or developed in the laboratory. Two Ae. aegypti colonies (Bora and Toahotu, established from the islands of Bora Bora and Tahiti, respectively) and two Ae. polynesiensis colonies (Tiaraunu from the atoll of Tetiaroa in the Society islands and "Aito" [BC9]) were used in this study. All colonies have been reared for several years, sometimes even decades (Ae. aegypti Bora), in the laboratory. The two Ae. aegypti colonies are known to be free of Wolbachia sp. whereas the Ae. polynesiensis Tiaraunu colonies are known to carry the bacterial endosymbiont 


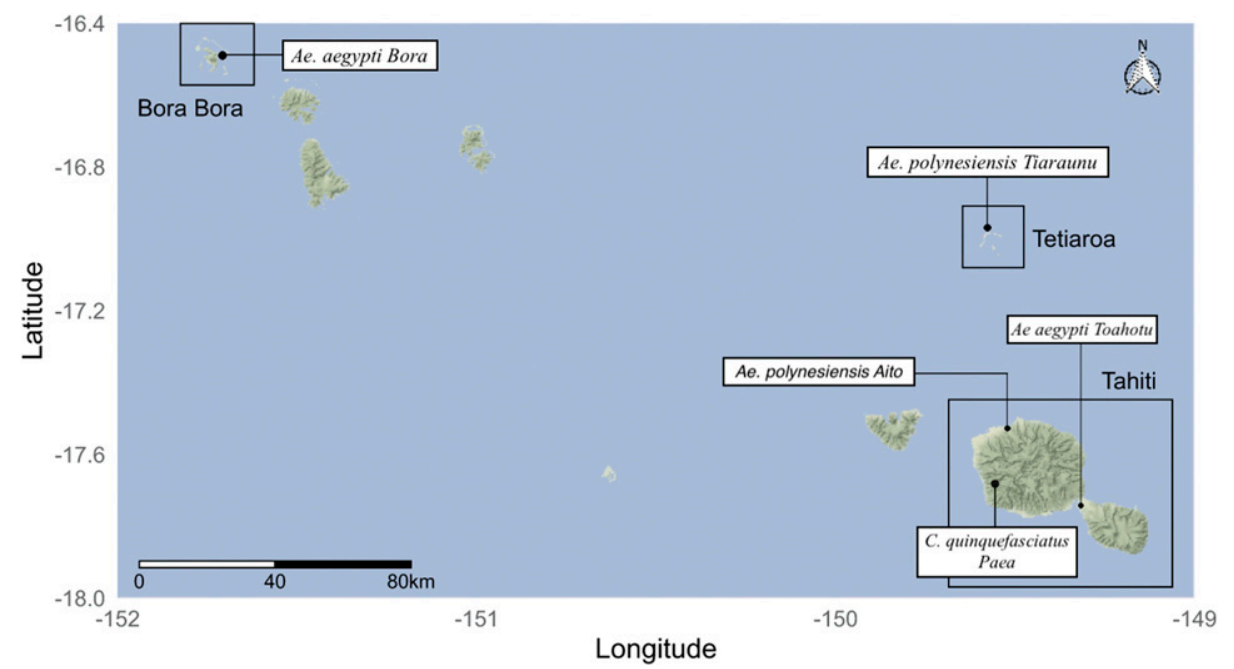

FIGURE 1. Map showing origin of mosquitoes (French Polynesia). The map was built using the ggmap package and the «terrain-background" map tile set provided by (C) Stamen Design, under a Creative Commons Attribution (CC BY 3.0) license. Data are provided by OpenStreetMap ${ }^{\circledR}$ (London, United Kingdom). This figure appears in color at www.ajtmh.org.

Wolbachia sp. clade A. ${ }^{36}$ The incompatible Ae. polynesiensis "Aito" (BC9) colony carries Wolbachia sp. clade $B .{ }^{31}$ The C. quinquefasciatus mosquitoes used in this study were collected in Paea, Tahiti, in September 2017 at the larval stage, and morphologically identified using morphological keys after they emerged as adults. ${ }^{37}$ Their infectious status regarding Wolbachia sp. was unknown.

All mosquitoes were stored frozen at $-80^{\circ} \mathrm{C}$ before shipping to IHU Méditerranée Infection laboratory in Marseille for further analyses. For additional experiments and controls, we used Aedes albopictus and Anopheles coluzzii reared in the insectarium of the laboratory in Marseille, as previously described. ${ }^{38}$

Sampling. A total of 500 mosquitoes belonging to the species Ae. aegypti from the Bora and Toahotu colonies, Ae. polynesiensis Tiauraunu and Aito [BC9] colonies, and wild specimens morphologically identified as $C$. quinquefasciatus from Paea (Tahiti, French Polynesia) were examined in this study. For each colony, an equal number of males and females (50 specimens each) were sampled. Details regarding each laboratory colony are presented in Table 1. For the An. coluzzii and $A$ e. albopictus species from the insectarium in Marseille,
24 specimens per species were included in the study (12 females and 12 males).

Sample preparation for MALDI-TOF MS. The legs of the mosquitoes were used for MALDI-TOF MS analyses, as previously described. ${ }^{20}$

Fresh mosquitoes from Marseille and thawed mosquitoes from French Polynesia were briefly washed in $70 \%$ ethanol, rinsed in distilled water, and then dried before dissection. All the legs of each mosquito were placed in Eppendorf tubes with glass powder, $15 \mu \mathrm{L}$ of $70 \%$ formic acid (Sigma, Lyon, France), and $15 \mu \mathrm{L}$ of $50 \%$ acetonitrile (volume to volume) (Fluka, Buchs, Switzerland). The samples were crushed using the Tissue Lyser instrument (Qiagen, Hilden, Germany) over three cycles at 30 movements per second for 60 seconds. ${ }^{39}$ The crushed samples were centrifuged at $200 \mathrm{~g}$ for 1 minute, and $1.5 \mu \mathrm{L}$ of the supernatant of each sample was deposited on a MALDI-TOF MS target plate (Bruker Daltonics, Wissembourg, France) in quadruplicate. Each spot was then recovered with 1.5 $\mu \mathrm{L}$ of $\mathrm{CHCA}$ matrix solution composed of saturated $\alpha$-cyano-4hydroxycynnamic acid (CHCA) (Sigma), 50\% acetonitrile (v/v), $2.5 \%$ trifluoroacetic acid (v/v) (Aldrich, Dorset, United Kingdom), and high performance liquid chromatography (HPLC) grade

TABLE 1

Results of mosquito sampling from French Polynesia, number of specimens used for the blind test and their identification score

\begin{tabular}{|c|c|c|c|c|c|c|c|c|c|}
\hline Species & Colony & Sex & $\begin{array}{l}\text { Wolbachia } \\
\text { supergroup }\end{array}$ & Origin & Number & MS & DB & BT & $\begin{array}{c}\text { Correct identification } \\
\text { [range log score value] (mean) }\end{array}$ \\
\hline \multirow[t]{4}{*}{ Aedes aegypti } & BORA & Males & None & BORA BORA* $^{*}$ & 48 & 48 & 0 & 48 & $48[1.8-2.4](2.2)$ \\
\hline & & Females & & & 48 & 48 & 0 & 48 & \\
\hline & TOAHOTU & Males & None & TAHITI* & 48 & 48 & 0 & 48 & $48[1.8-2.3](2.1)$ \\
\hline & & Females & & & 48 & 48 & 0 & 48 & \\
\hline \multirow[t]{4}{*}{ Aedes polynesiensis } & AITO [BC9] & Males & B & ILM-TAHITI* & 48 & 48 & 1 & 47 & $47[2-2.7](2.5)$ \\
\hline & & Females & B & & 48 & 48 & 1 & 47 & \\
\hline & TIARAUNU & Males & A & TETIAROA* & 48 & 48 & 0 & 48 & $48[1.8-2.7](2.5)$ \\
\hline & & Females & A & & 48 & 48 & 0 & 48 & \\
\hline \multirow[t]{3}{*}{ Culex quinquefasciatus } & PAEA & Males & ND & TAHITI† & 48 & 48 & 10 & 38 & 38 [1.9-2.6] (2.3) \\
\hline & & Females & & & 48 & 48 & 2 & 46 & \\
\hline & & & & & 480 & & & & \\
\hline
\end{tabular}

$\mathrm{ND}=$ not defined. The bold values represent the number of samples correctly identified with MALDI-TOF MS.

* Laboratory colonies.

†Field-collected specimen, MS = number of specimens included in the study and analyzed using mass spectrometry; DB = number of specimens entered in the database; BT = number of specimens used for blind test analyses. 
water. ${ }^{20,39}$ After drying for several minutes at room temperature, the target plate was placed in the Microflex LT MALDI-TOF mass spectrometer device (Bruker Daltonics) for analysis.

MALDI-TOF MS parameters. Protein mass profiles were generated using a Microflex LT MALDI-TOF mass spectrometer (Bruker Daltonics), with detection in the linear positive-ion mode at a laser frequency of $50 \mathrm{~Hz}$ within a mass range of 2-20 kDa. The acceleration voltage was $20 \mathrm{kV}$, and the extraction delay time was $200 \mathrm{~ns}$. Each spectrum corresponded to ions obtained from 240 laser shots performed in six regions of the spot and automatically acquired using the AutoXecute method of the FlexControl v. 2.4 software (Bruker Daltonics). The spectrum profiles were viewed using FlexAnalysis v. 3.3 software and exported to ClinProTools v. 2.2 and MALDI-Biotyper v. 3.0 (Bruker Daltonics) for data processing (smoothing, baseline subtraction, and peak picking), GelView and principal component analysis (PCA), and main spectrum profile (MSP) creation. ${ }^{39}$

MALDI-TOF MS identification of mosquitoes. The quality of the spectra was visually validated using the FlexAnalysis v. 3.3 software by assessing general intensity of the spectra $\left(10^{4}\right.$, ideally), the smoothness of the peaks, a flat baseline, and reproducibility compared with other spectra of the same mosquito species. Spectra reproducibility of legs from all mosquito specimens was evaluated by comparing the average spectra of each sample within its respective species using the GelView tool of ClinProTools 2.2 software (Bruker Daltonics). The MSPs were produced based on an algorithm taking information on the peak position, intensity, and frequency. Consistency of MS profiles between males and females of the same species was evaluated by comparison of MS profiles with ClinProTools 2.2 software. Using the same software, the two average spectra of all males and all females, respectively, were superimposed to highlight distinctive peaks. ${ }^{40}$ The distinction between two colonies of the same species was evaluated on spectra of female mosquitoes by PCA using ClinProTools software.

The spectra of each mosquito specimen were queried against the existing homemade reference database using MALDI-Biotyper software v. 3.0 (Bruker Daltonics). ${ }^{41}$ The arthropod homemade MS database was upgraded with reference spectra of two specimens of Ae. polynesiensis of both sexes, as spectra of this species were absent in our database; and two reference spectra of $C$. quinquefasciatus Paea after confirmation by molecular biology. After a first blind test against this upgraded database, 10 spectra of $C$. quinquefasciatus Paea specimens with good spectra and log score values (LSVs) $<1.8$ were subjected to molecular identification and then added to the in-laboratory homemade MS database. A total of 14 MSPs were created using the four high-quality spectra of 14 mosquitoes. To visualize the similarity between MS profiles, appraise the diversity of included samples, and therefore assist with selecting relevant reference spectra, a dendrogram of C. quinquefasciatus spectra was created using the dendrogram function of MALDI Biotyper, version 3.0. The dendrograms are based on the results of a composite correlation index (CCl) matrix. Composite correlation indexs are calculated by dividing spectra into intervals and comparing these intervals across a data set. The composition of correlations of all intervals provides the $\mathrm{CCl}$, which is used as a parameter for defining the distance between the spectra. A CCl match value of one represents complete correlation, whereas a CCI match value of zero represents a lack of correlation. ${ }^{42}$ The spectra of all specimens, except those used to upgrade the database, were queried against the in-laboratory homemade MS database (Supplemental Figure 1A and B). The level of identification was determined using LSVs given by MALDI-Biotyper v. 3.0 software, corresponding to a matched degree of signal intensities of mass spectra of the query and the reference spectra. Log score values range from zero to three and allow a good evaluation of the reproducibility between a queried spectrum and a reference spectrum, as it is the result of thorough comparison of peak position and intensity between those two spectra (MALDI-BioTyper Help, Bruker Daltonics). An LSV was thus obtained for each of the four spectra of the blind-tested samples. For each sample, the spectrum with the highest LSVs was selected for reliable identification.

MALDI-TOF MS biomarker mass set. To determine the differential peaks between Ae. aegypti Bora and Ae. aegypti Toahotu, a subset of 22 high-quality MS spectra of each colony were loaded into ClinProTools 2.2 software. The software was used to generate a peak list for each group in the $2-20 \mathrm{kDa}$ mass range and to identify discriminating peaks. The parameter settings in ClinProTools 2.2 software for spectra preparation were as follows: a resolution of 800 ppm, a noise threshold of 3.00 (intensity arbitrary unit), a maximum peak shift of 800 ppm, and a match to calibration peaks of $20 \%$. For the peak calculation, peak picking was performed on single spectra with a signal-to-noise threshold of 2.00 and an aggregation of 800 ppm. Manual inspection and validation of the selected peaks by the operator gave a "recognition capability" $(\mathrm{RC})$ value together with the highest "cross-validation" (CV) value. These values reflect the ability to distinguish the different spectra groups based on the chosen discriminatory peaks. Genetic algorithm settings are manually optimized to provide the highest $\mathrm{RC}$ and $\mathrm{CV}$ values with the smallest number of peaks.

Molecular identification of wild mosquitoes. For each mosquito, the head and thorax were transferred to a $1.5-\mathrm{mL}$ Eppendorf tube containing $180 \mu \mathrm{L}$ of lysis buffer and $20 \mu \mathrm{L}$ of proteinase K (Qiagen) and incubated at $56^{\circ} \mathrm{C}$ overnight. DNA extraction was performed with EZ1 Advanced XL Robot (Qiagen) using the EZ1 DNA Tissue Kit (Qiagen) in accordance with the manufacturer's instructions. DNA from each sample was eluted with $100 \mu \mathrm{L}$ Qiagen Tris-EDTA buffer and immediately used or stored at $-20^{\circ} \mathrm{C}$.

Mosquito specimens morphologically identified as C. quinquefasciatus, which spectra gave LSVs $<1.8$, despite being of good quality, were submitted for molecular identification. For this purpose, a fragment of the acetylcholinesterase-2 gene was amplified by PCR and sequenced. ${ }^{43}$ The amplified products were subjected to electrophoresis on agarose gel and viewed using ultraviolet on a Gel Doc System (Bio-Rad, Hercules, CA). The PCR products of the positive samples were purified and sequenced using the same primers, BigDye version 1-1 Cycle Sequencing Ready Reaction Mix (Applied Biosystems, Foster City, CA), and an ABI 3100 automated sequencer (Applied Biosystems). The sequences were assembled and analyzed using the ChromasPro software (version 1.34) (Technelysium Pty. Ltd., Tewantin, Australia) and the NCBI BLAST website (http://blast.ncbi.nlm.nih.gov).

\section{RESULTS}

MALDI-TOF MS analysis and blind test validation. The MS spectra of 192 Ae. aegypti specimens of both Ae. aegypti Bora and $A$ e. aegypti Toahotu colonies were queried against 
our in-laboratory database. Built over the years, this database already contained Ae. aegypti reference spectra obtained from specimens from Senegal, and also Réunion Island and Mayotte, two overseas regions of France in the Indian Ocean. ${ }^{20}$ The blind test performed against this database revealed an accurate identification of the 192 spectra of $A e$. aegypti from French Polynesia, matching reference Ae. aegypti spectra from other countries. Their LSVs ranged from 1.8 to 2.4 (Table 1). MALDI-TOF MS was also applied to Ae. polynesiensis, a species not yet available in our arthropod MS database. The visual evaluation of the spectra from this species using FlexAnalysis and ClinProTools software revealed that all specimens provided highly reproducible spectra of excellent quality (Figure 2). One male and one female spectra of Ae. polynesiensis were selected based on the global intensity and overall quality of the spectra to upgrade our database. The spectra of 190 samples of Ae. polynesiensis Aito [BC9] and Ae. polynesiensis Tiaraunu subsequently underwent a blind comparison against this upgraded database. All specimens were successfully identified as Ae. polynesiensis with LSVs ranging from 1.8 to 2.7 (Table 1) based on the two specimens recently added to the in-laboratory database.

MALDI-TOF MS analysis of wild specimens of C. quinquefasciatus collected in Paea (Tahiti) revealed good quality and reproducible spectra using FlexAnalysis and ClinProTools software. The first blind test against the reference database containing spectra of $C$. quinquefasciatus from Senegal, Mali, Australia, and Chad, and two newly added reference spectra from Paea (French Polynesia) showed that $75 / 94(79.8 \%)$ of the samples morphologically identified as C. quinquefasciatus had LSVs $>1.8$ and 19 of 94 spectra had LSVs $<1.8$. All these 19 low-LSV spectra were of good quality. Ten specimens among the 19 outliers were selected for molecular identification. These 10 specimens were successfully identified as C. quinquefasciatus, with $99.6-100 \%$ identity with the reference sequences available in GenBank (GenBank accession number: FJ416029). The spectra of these molecularly identified specimens were then added to the MALDI-TOF MS database, which was then used to test the remaining nine specimens. Blind test queries resulted in correct identification of all 84 spectra of $C$. quinquefasciatus Paea specimens, and included the nine specimens with previous ambiguous identification, with LSV ranging from 1.9 to 2.6 (mean: 2.3) (Table 1).

MALDI-TOF MS analysis revealed high reproducibility of male and female $A$ e. aegypti Bora and $A$ e. aegypti Toahotu MS spectra (Figure $3 A$ and $B$ ). The superimposition of the average MS profiles of the males and females of each colony showed discriminatory peaks between the two groups, with a higher intensity within the females for some peaks (Figure $3 \mathrm{C}$ ). The similarity of the MS profiles was assessed using the PCA function integrated in the ClinProTools software, based on peak information such as position and intensity. The PCA

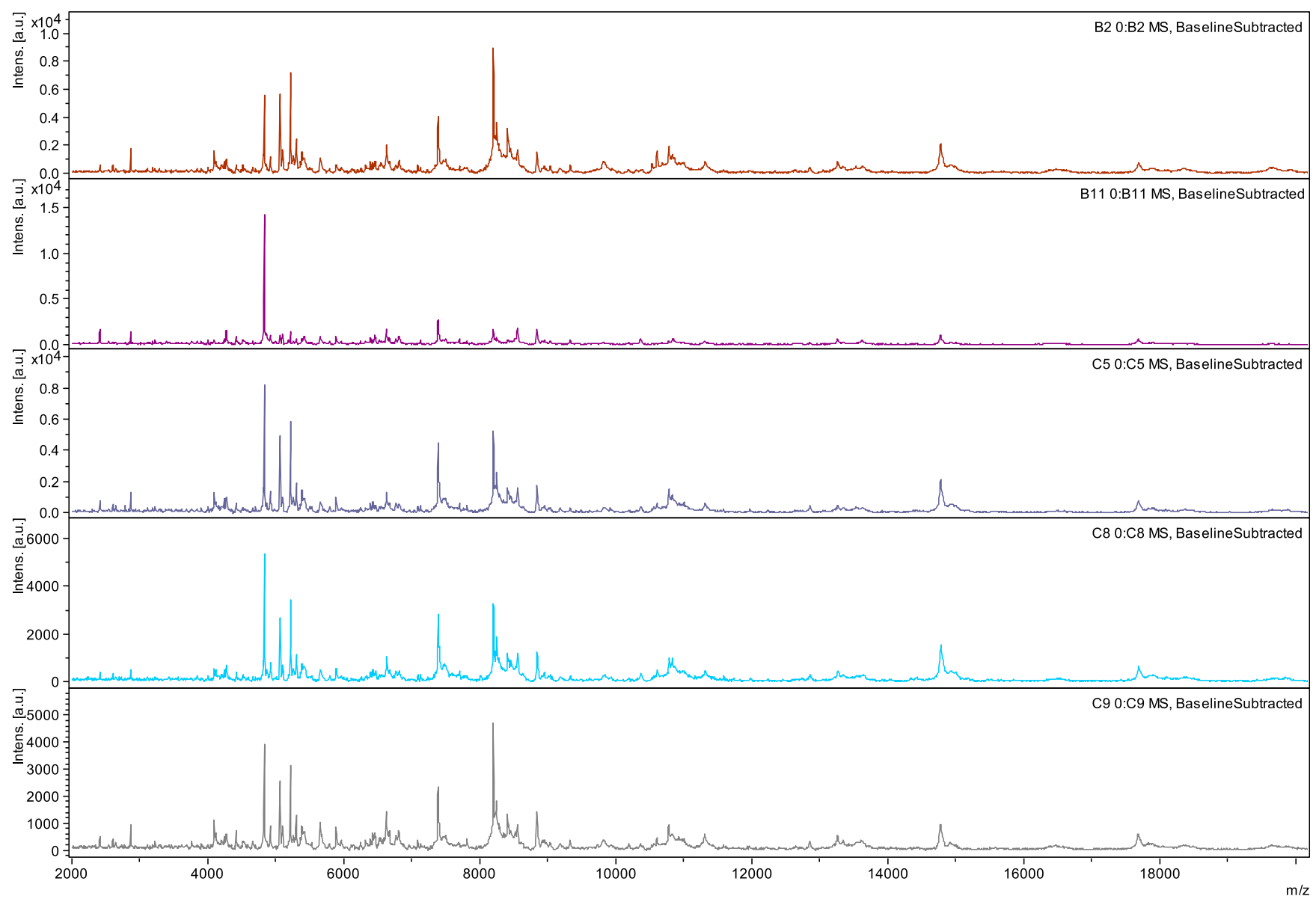

FIGURE 2. Representative MS profiles of Ae. polynesiensis Aito [BC9] using Flex analysis 3.3 software. a.u. $=$ arbitrary units; $\mathrm{m} / \mathrm{z}=\mathrm{mass}$-to-charge ratio. This figure appears in color at www.ajtmh.org. 

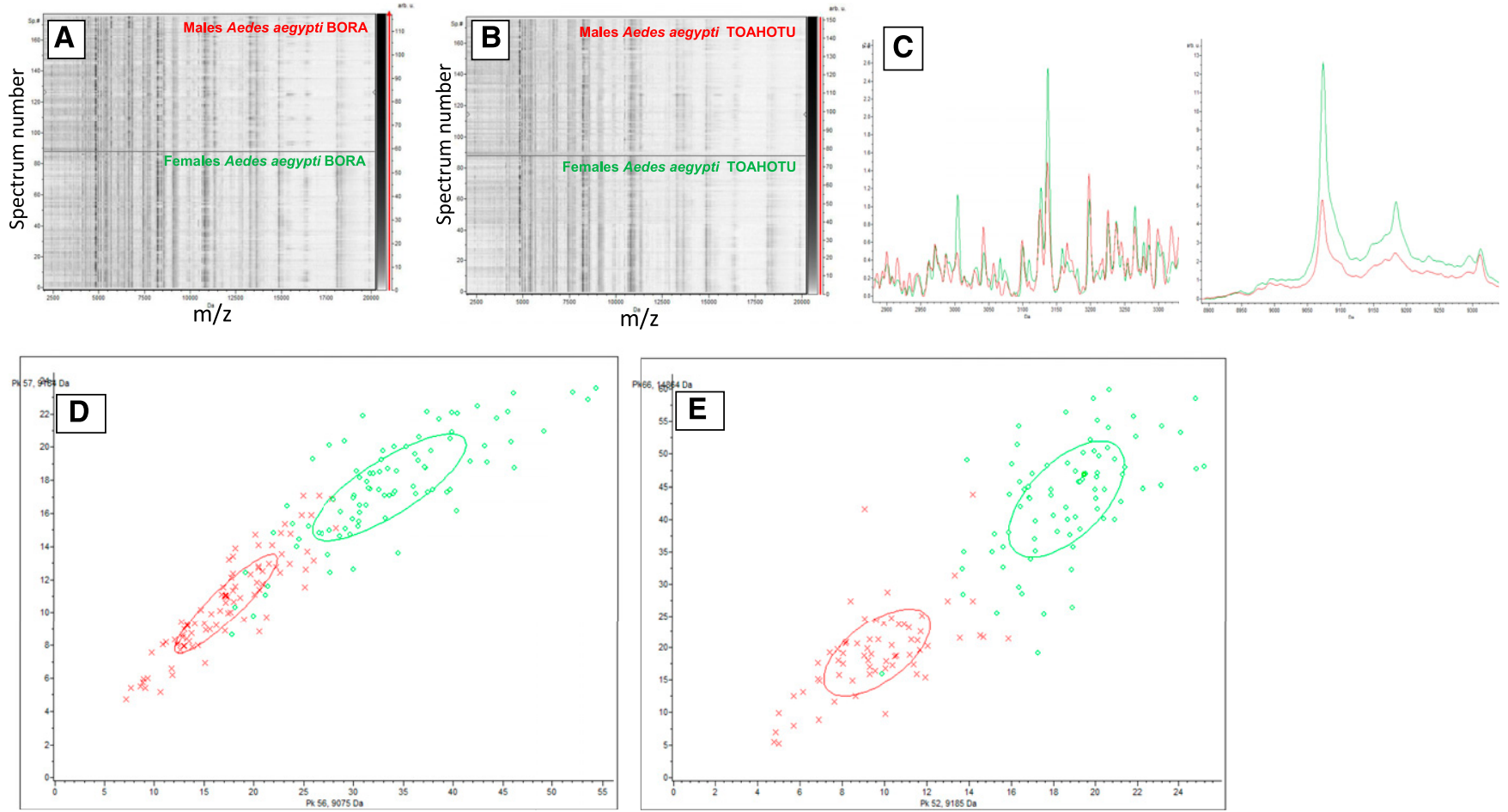

FIGURE 3. Comparison of male and female Aedes aegypti mosquitoes MALDI-TOF MS spectra using ClinProTools 2.2 software. Gel view representation of MS profiles of male/female Ae. aegypti Bora (A) and Ae. aegypti Toahotu (B) mosquitoes. (C) Superimposition of average MS profiles male (red) and female (green) mosquitoes. Principal component analysis (PCA) of male (red) and females (green) Ae. aegypti Bora (D) and Ae. aegypti Toahotu (E) MS spectra. m/z, mass-to-charge ratio. This figure appears in color at www.ajtmh.org.

presented in (Figure 3D and E) revealed a difference between males and females of the same colony. The similar difference was later observed also for specimens Ae. albopictus and $A n$. coluzzii from laboratory colonies of Marseille (Figure $4 A$ and $B$ ). Principal component analysis was also used to highlight this difference using male and female spectra of Ae. polynesiensis Aito [BC9] and Ae. polynesiensis Tiaraunu (Figure 5A and $\mathrm{B}$ ).

To highlight spectrum differences between the two Ae. aegypti colonies, namely, Bora and Toahotu, their spectra were visualized as a GelView using the ClinProTools software.
GelView showed good reproducibility of the spectra for both colonies (Figure 6A). Female spectra were selected for further analyses. With a slightly better quality than male spectra, they would allow identification of subtle variations. A difference in the profiles between female Ae. aegypti Bora and Ae. aegypti Toahotu was evidenced by a PCA, as shown in Figure 6B. The genetic algorithm tool of ClinProTools software was used to better identify the discriminating peaks between the same female Ae. aegypti Bora and Ae. aegypti Toahotu. To do this, a subset of 22 high quality spectra from female mosquito each
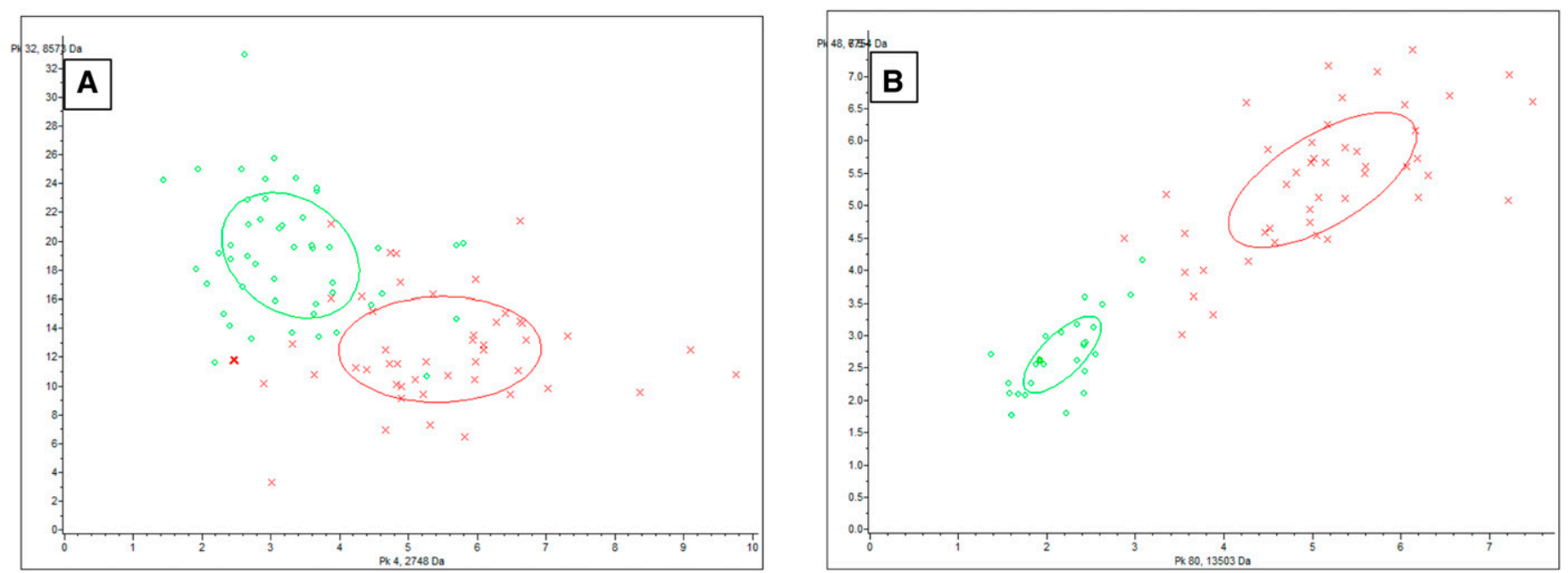

FIGURE 4. Principal component analysis (PCA) of (A) male/female Aedes albopictus and (B) male/female Anopheles coluzzii using ClinProTools 2.2 software. This figure appears in color at www.ajtmh.org. 

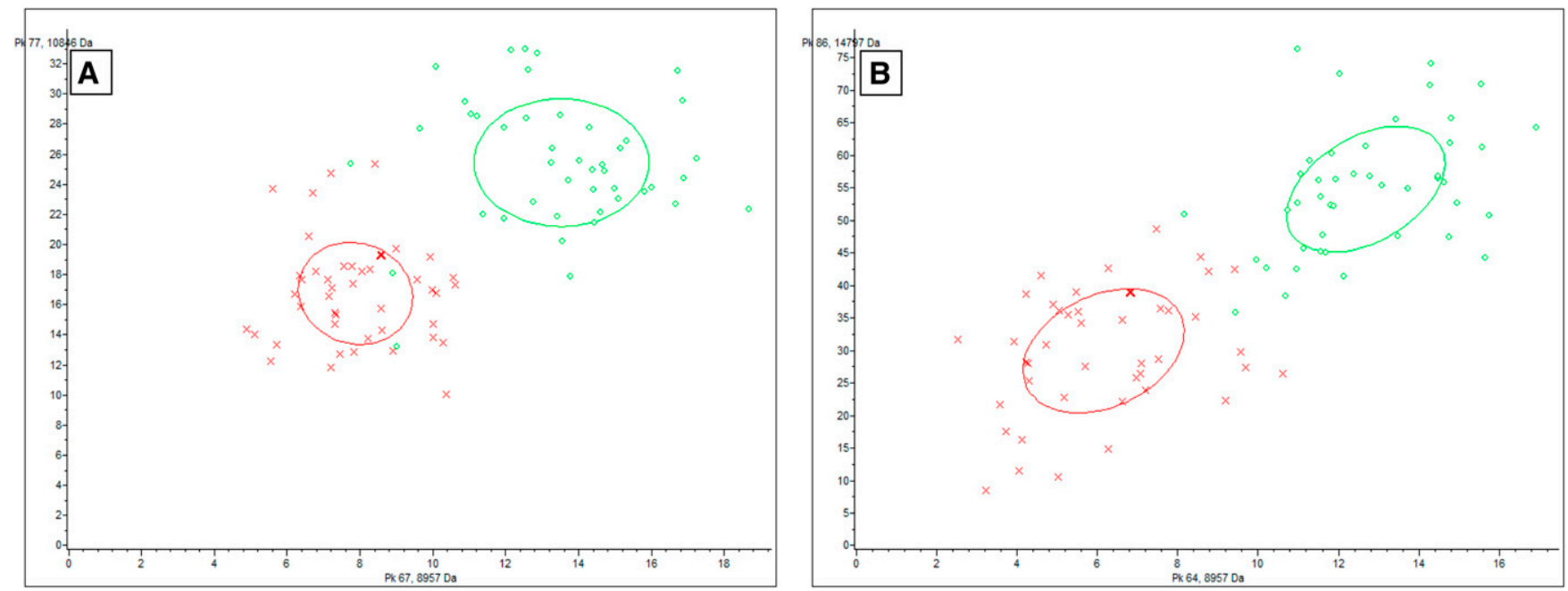

FIGURE 5. Principal component analysis (PCA) of male (red) and female (green) Aedes polynesiensis Aito [BC9] (A) and Ae. polynesiensis Tiaraunu mosquitoes (B) using ClinProTools 2.2 software. This figure appears in color at www.ajtmh.org.

colony was loaded into the ClinProTools software, and this analysis revealed nine peak masses (Table 2) that allowed optimal discrimination of the two colonies, with $\mathrm{RC}$ and $\mathrm{CV}$ values of $98.2 \%$ and $85.0 \%$, respectively. A distinction was also noted between the female Ae. polynesiensis Aito [BC9] and Ae. polynesiensis Tiaraunu spectra (Figure 7).

\section{DISCUSSION}

In this study, Ae. aegypti specimens from French Polynesia were correctly identified by MALDI-TOF MS analysis with LSVs ranging from 1.8 to 2.4 based on Ae. aegypti reference spectra from Senegal, Mayotte, and Réunion Island. Proof-ofconcept studies for the identification of arthropods by MALDITOF MS analysis set up a threshold LSV at 1.8 for accurate identification of the species. ${ }^{20,41}$ Our results on Ae. aegypti specimens from French Polynesia suggest that despite the geographical variability observed between populations of a same mosquito species, a successful identification can be achieved based on reference spectra from another country. Aedes polynesiensis was absent from our database, which was upgraded using two specimens of that species. All queried specimens were identified with high LSVs ranging from 1.8 to 2.7. As for C. quinquefasciatus, all specimens were correctly identified when queried against our database containing spectra of $C$. quinquefasciatus from Australia, Chad, Senegal, Mali, and Paea (French Polynesia). Some specimens however yielded LSVs lower than 1.8. This might be attributed to the known spectrum variability of $C$. quinquefasciatus specimens from different geographical areas. This had been previously described in previous studies attempting to identify Culex spp. from Africa using MALDI-TOF MS. ${ }^{41,44}$ To cover the diversity of these wild $C$. quinquefasciatus specimens, reference spectra from 12 mosquitoes were selected through a dendrogram; their identification was confirmed by molecular biology before adding them to the database. The addition of
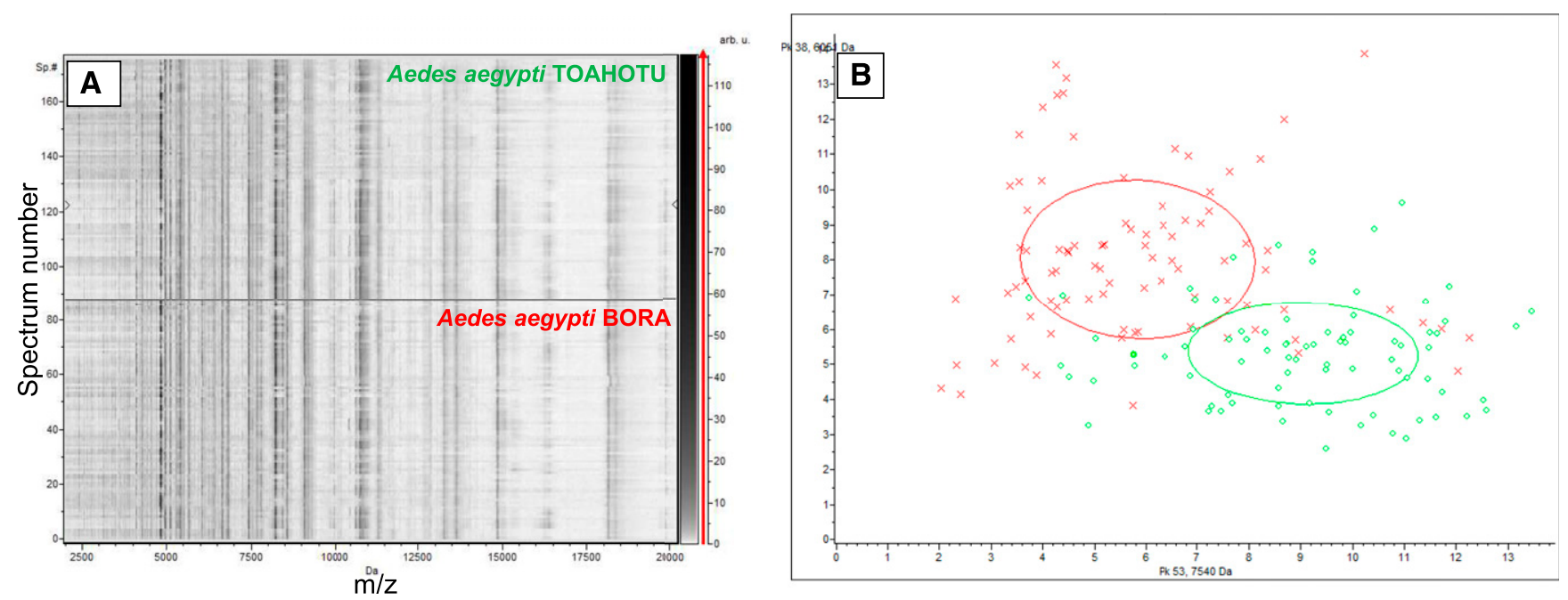

FIGURE 6. Comparison of Aedes aegypti Bora and Ae. aegypti Toahotu MALDI-TOF MS spectra using ClinProTools 2.2 software. (A) GelView representation of MS profiles of Ae. aegypti Toahotu (green) and Ae. aegypti Bora (red) mosquitoes by the view gel tool. (B) Principal component analysis (PCA) of the Ae. aegypti Bora (red) and Toahotu (green) spectra. $\mathrm{m} / \mathrm{z}=$ mass-to-charge ratio. This figure appears in color at www.ajtmh.org. 
TABLE 2

Determination of peak masse distinguishing Ae. aegypti Bora from Ae. aegypti Toahotu

\begin{tabular}{lll}
\hline Mass $(\mathrm{m} / \mathrm{z})(\mathrm{Da})$ & Ae. aegypti Bora & Ae. aegypti Toahotu \\
\hline 3,376 & Decreased & Increased \\
4,573 & Increased & Decreased \\
5,889 & Decreased & Increased \\
6,241 & Decreased & Increased \\
7,524 & Increased & Decreased \\
8,106 & Decreased & Increased \\
9,464 & Increased & Decreased \\
10,109 & Decreased & Increased \\
11,113 & Increased & Decreased \\
\hline Ae. aegypti=Aedes aegypti. & &
\end{tabular}

these 12 French Polynesia C. quinquefasciatus reference spectra led to an accurate identification of all remaining specimens with higher LSVs. This confirms the necessity on having a comprehensive database for a reliable identification of mosquito species. Although more work is needed to determine a definitive cutoff for MALDI-TOF MS arthropod accurate identification, our study highlights the need to include a large diversity of specimens within the same species in the database, at least for some species. The intraspecies variability of $C$. quinquefasciatus justifies the addition of this many spectra of specimens from Paea in the database. Previous studies on bedbugs ${ }^{45}$ highlighted that more reference spectra are needed for correct identification in the case of intragroup variability.

The spectra of Ae. polynesiensis were on the other hand highly reproducible, and therefore, all specimens were properly identified based on two reference spectra only. In a previous study, highly reproducible spectra from Australian mosquitoes were also successfully identified using one to three reference spectra only. ${ }^{46}$ The need to have a universal database was highlighted by Raharimalala et al. ${ }^{23}$ It should also be noted that currently there is no MALDI-TOF MS universal online arthropod database. We are however in the process of building an open access database such as the ones currently available for mycological ${ }^{47}$ and bacterial ${ }^{48}$ identification. Nevertheless, the spectra files can be easily transferred to any Bruker MALDI-TOF MS device, so our database is already accessible through collaborative projects.

This study also confirmed the suitability of freezing mosquito samples when they are meant to be later identified using MS. We observed indeed that all the perfectly frozen mosquito samples revealed very good quality spectra. This observation is in line with previous works on sand flies, mosquitoes, and ticks which proposed that the best MALDI-TOF MS results are to date obtained if the specimens are kept frozen. ${ }^{28,39,49}$ This does not preclude other methods of storing mosquito samples such as storage in $70 \%$ ethanol and in silica gel, which are generally used in the field because this is easier and cheaper to

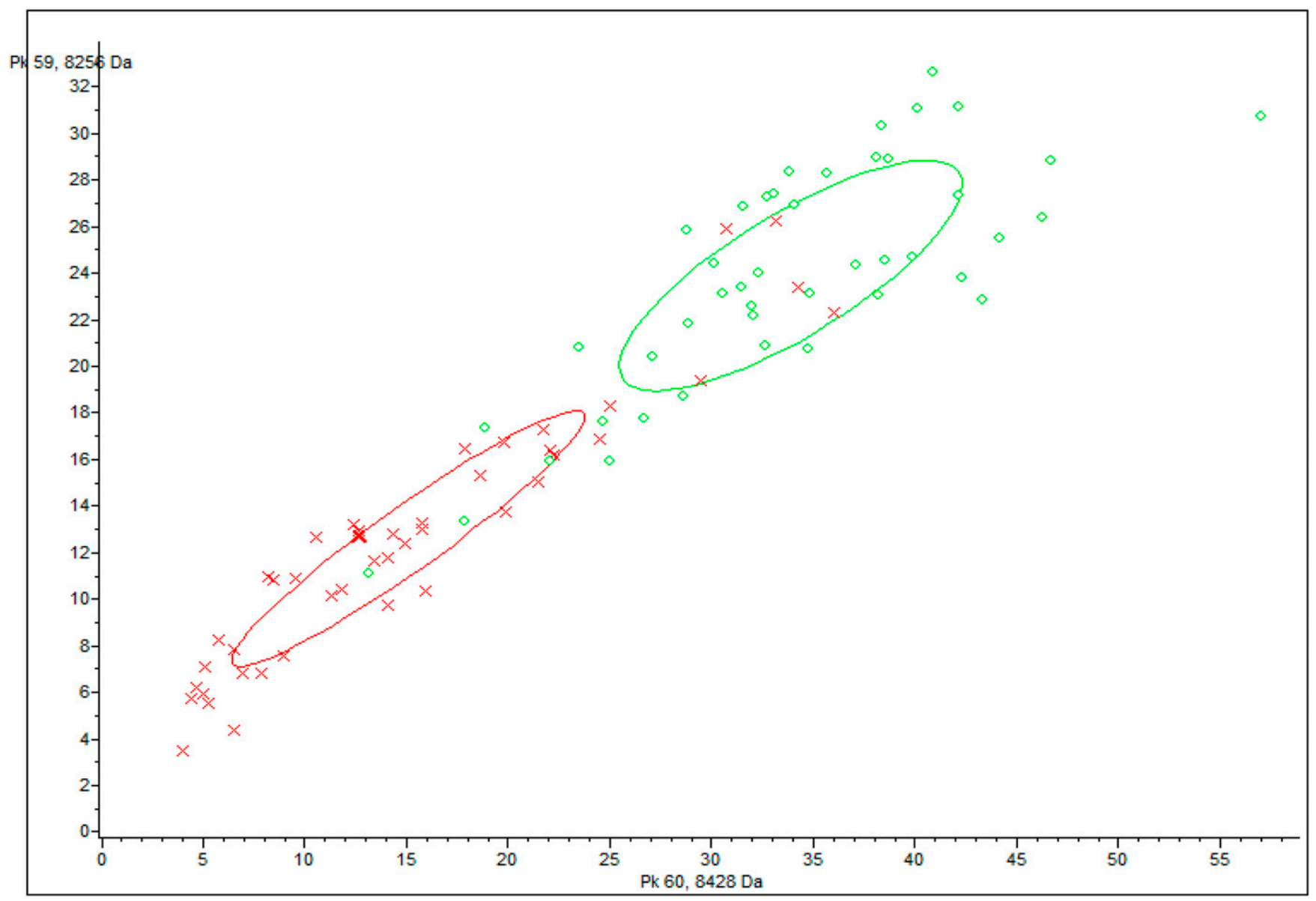

FIGURE 7. Principal component analysis (PCA) of Aedes polynesiensis Aito [BC9] (green) and Tiaraunu (red) mosquito spectra using ClinProTools 2.2 software. This figure appears in color at www.ajtmh.org. 
perform. However, these preservation methods have some limitations for MALDI-TOF MS analysis because they can lead to a decrease in the intensity and quality of the MS spectra of the samples. It has been reported that the preservation of a specimen for a long time in $70 \%$ ethanol can have negative effects on the quality of the MS spectra. ${ }^{25,29,39}$ This might be resolved in the future by improving the protocols of sample preparation prior to MALDI-TOF MS, hence the need to not only adjust the protein extraction protocol of the specimen according to the sample storage method but also have a specific database for each protocol. ${ }^{39,41}$

Since its first application in medical entomology, the use of MALDI-TOF MS has been increasingly diverse. The precision of the tool has allowed the robust identification of blood meals, ${ }^{38,44}$ and the differentiation of arthropods infected or not by selected microorganisms. ${ }^{40,45}$ Here, we assessed for the first time the ability of MALDI-TOF MS to determine the sex of mosquitoes. Morphological criteria have always been used to easily differentiate between male and female mosquitoes ${ }^{50}$; this differentiation between the two sexes is a key point in entomological studies and therefore vector control programs because it is the females that feed on blood and transmit pathogens. ${ }^{51}$ Although the differentiation between males and females can be made very easily morphologically and with the naked eye by mosquito experts or trained people, MALDI-TOF MS allows us to quickly obtain both the identification of the mosquito and the sex without any prior training. It is known that the protein profile is different between females and males ${ }^{52}$; this is confirmed here using MALDI-TOF MS and has also been demonstrated using wild specimens and specimens from laboratory colonies from French Polynesia and Marseille. Mosquitoes feed on nectar, but only females are hematophagous. They require blood meals that allow them to assimilate more protein, which is essential for vitellogenesis. $^{52}$ This could then explain the variability of their protein content compared with males. We have also noticed that the difference between males and females is more visible in $\mathrm{Ae}$. aegypti Toahotu than in Ae. aegypti Bora, but we have not yet found explanations for this phenomenon.

We also show herein that MALDI-TOF MS analyses distinguished female mosquito colonies of the same species but from different geographical origin. Using female specimens, Ae. aegypti Bora and Ae. aegypti Toahotu colonies were clearly segregated into two clusters in a PCA, and so were the two Ae. polynesiensis colonies (Aito [BC9] and Tiaraunu). To show this differentiation between colonies of the same species, female specimens were used as they provided slightly more homogeneous spectra than the males. As illustrated by the GelView (Figure 3A and B and Supplemental Figure 2A-C), the difference between the male and female spectra is minimal, invisible with the naked eye, without further analyses such as average spectra superimposition. We have also shown that the major observed difference between male and female mosquitoes is based on the intensity of some protein peaks. We can therefore expect that the difference observed between female colonies, which is based on differential peaks and not peak intensity, could be found in males as well. This shows that there is a difference in the protein composition between specimens of the same species from different geographical areas, even when reared in the same laboratory conditions. The impact of the duration of laboratory rearing - and therefore the number of generations-is not known. It could therefore be assumed that there are environmental or biological factors which differ in each geographic area, and which could affect the development of species or even their protein composition for several generations.

We also noticed that the differentiation between the $A e$. polynesiensis Aito [BC9] and Ae. polynesiensis Tiaraunu colonies was more pronounced than that between the Ae. aegypti Bora and Ae. aegypti Toahotu colonies. The two Ae. polynesiensis colonies are infected by two different supergroups of Wolbachia sp., that is, supergroup A for the Ae. polynesiensis Tiaraunu and supergroup B for the Ae. polynesiensis Aito [BC9] colony. It could be hypothesized that this stronger differentiation between these two colonies could be due to this additional factor, that is, the presence of the two different supergroups of Wolbachia sp. However, we cannot support this hypothesis because of the existing bias from geographical origin of the two colonies. This is a limit to our study because to truly confirm whether MALDI-TOF MS is able to distinguish Wolbachia-infected mosquitoes from noninfected ones, we would need specimens of the same species, from the same location, and infected or not by the same supergroups of Wolbachia sp.

Ultimately, this work confirmed that the MALDI-TOF MS is a fast, accurate, and cost-effective tool to identify field mosquitoes using only their legs. ${ }^{22-24}$ It also showed for the first time that this tool was able to distinguish not only male mosquitoes from females within the same colony but also between different colonies of the same species according to their geographical distribution. The MALDI-TOF MS tool could thus make an important contribution to vector control programs.

Received January 14, 2020. Accepted for publication January 19, 2021.

Published online March 22, 2021.

Note: Supplemental information, table, and figures appear at www. ajtmh.org.

Acknowledgments: We thank Adama Zan Diarra for his kind advice with sequencing, Handy Dahmana for rearing the Aedes albopictus, and Michel Cheong Sang and Jérôme Marie, respectively, for rearing and collecting the French Polynesia mosquitoes used in this study.

Financial support: This study was supported by the Institut HospitaloUniversitaire (IHU) Méditerranée Infection, the National Research Agency under the program "Investissements d'avenir ", reference ANR-10-IAHU-03, the Région Provence Alpes Côte d'Azur, and European funding FEDER PRIMI.

Authors' addresses: Fatou Kiné Fall, Aix Marseille University, IRD, APHM, SSA, VITROME, Marseille, France, IHU Méditerranée Infection, Marseille, France, and Campus International IRD-UCAD de I'IRD, Dakar, Senegal, E-mail: fakifa22@hotmail.fr. Maureen Laroche and Philippe Parola, Aix Marseille University, IRD, AP-HM, SSA, VITROME, Marseille, France, and IHU Méditerranée Infection, Marseille, France, E-mails: mlaroche@kemri-wellcome.org and philippe.parola@univamu.fr. Hervé Bossin, Medical Entomology Laboratory, Institut Louis Malardé, Papeete, Tahiti, French Polynesia, E-mail: hbossin@ilm.pf. Didier Musso, Aix Marseille University, IRD, AP-HM, SSA, VITROME, Marseille, France, and SELAS Eurofins Labazur Guyane, Cayenne, French Guiana, E-mail: dmusso12345@gmail.com.

\section{REFERENCES}

1. World Health Organization, 2017. Vector-Borne Diseases. Geneva, Switzerland: WHO. Available at: http://www.who.int/fr/newsroom/fact-sheets/detail/vector-borne-diseases. Accessed October 30, 2019.

2. Institut Louis-Malardé, 2019. Fiche-Thematique-Moustique1.pdf. Available at: https://www.ilm.pf/wp-content/uploads/ 2019/11/Fiche-thematique-moustique-1.pdf. Accessed March $18,2020$. 
3. Musso D, Rodriguez-Morales AJ, Levi JE, Cao-Lormeau VM, Gubler DJ, 2018. Unexpected outbreaks of arbovirus infections: lessons learned from the Pacific and tropical America. Lancet Infect Dis 18: e355-e361.

4. Cao-Lormeau VM, Roche C, Aubry M, Teissier A, Lastere S, Daudens E, Mallet H-P, Musso D, Aaskov J, 2011. Recent emergence of dengue virus serotype 4 in French Polynesia results from multiple introductions from other south Pacific islands. PLoS One 6: e29555.

5. Cao-Lormeau VM, Roche C, Musso D, Mallet HP, Dalipanda T, Dofai A, Nogareda F, Nilles EJ, Aaskov J, 2014. Dengue virus type 3, south Pacific islands, 2013. Emerg Infect Dis 20: 1034-1036.

6. Aubry $\mathrm{M}$ et al., 2015. Chikungunya outbreak, French Polynesia, 2014. Emerg Infect Dis 21: 724-726.

7. Musso D et al., 2018. Zika virus in French Polynesia 2013-14: anatomy of a completed outbreak. Lancet Infect Dis 18: e172-e182.

8. Dupon JF, Bonvallot J, Vigneron E, 1993. Atlas de la Polynésie française. Paris, France: Editions de l'ORSTOM.

9. Guillaumot L, 2005. Arboviruses and their vectors in the Pacific-status report. Pac Health Dialog 12: 45-52.

10. Richard V, Paoaafaite T, Cao-Lormeau VM, 2016. Vector competence of French polynesian Aedes aegypti and Aedes polynesiensis for zika virus. PLoS Negl Trop Dis 10: e0005024.

11. Richard V, Cao-Lormeau VM, 2019. Mosquito vectors of arboviruses in French Polynesia. New Microbes New Infect 31: 100569.

12. Belkin JN, 1962. The Mosquitoes of the South Pacific: (Diptera, Culicidae). Oakland, CA: University of California Press.

13. World Health Organization, 2003. Entomologie du paludisme et contrôle des vecteurs. Geneva, Switzerland: WHO. Available at: https://apps.who.int/iris/bitstream/handle/10665/68376/WHO_ CDS_CPE_SMT_2002.18_Rev.1_Partiel.pdf;jsessionid= C510CBC98BA49D9EBD008017E9A41AC8? sequence $=1$. Accessed August 7, 2019.

14. Hervy JP, Le Goff G, Geoffroy B, Hervé J-P, Manga L, Brunhes J, 1998. The Anopheline Mosquitoes of the Afrotropical Region: An Identification and Training Software. Paris, France: ORSTOM. Available at: http://www.documentation.ird.fr/hor/fdi: 010014161. Accessed August 7, 2019.

15. Kent RJ, Deus S, Williams M, Savage HM, 2010. Development of a multiplexed polymerase chain reaction-restriction fragment length polymorphism (PCR-RFLP) assay to identify common members of the subgenera Culex (Culex) and Culex (phenacomyia) in Guatemala. Am J Trop Med Hyg 83: 285-291.

16. Laroche $M$ et al., 2017. Medical entomology: a reemerging field of research to better understand vector-borne infectious diseases. Clin Infect Dis 65: S30-S38.

17. Higa Y, Toma T, Tsuda Y, Miyagi I, 2010. A multiplex PCR-based molecular identification of five morphologically related, medically important subgenus Stegomyia mosquitoes from the genus Aedes (Diptera: Culicidae) found in the Ryukyu Archipelago, Japan. Jpn J Infect Dis 63: 312-316.

18. Lv J et al., 2014. Assessment of four DNA fragments (COI, 16S rDNA, ITS2, 12S rDNA) for species identification of the Ixodida (Acari: Ixodida). Parasit Vectors 7: 93.

19. Yssouf A, Almeras L, Raoult D, Parola P, 2016. Emerging tools for identification of arthropod vectors. Future Microbiol 11: 549-566.

20. Yssouf A et al., 2013. Matrix-assisted laser desorption ionization-time of flight mass spectrometry: an emerging tool for the rapid identification of mosquito vectors. PLoS One 8: e72380.

21. Seng P, Rolain JM, Fournier PE, La Scola B, Drancourt M, Raoult D, 2010. MALDI-TOF-mass spectrometry applications in clinical microbiology. Future Microbiol 5: 1733-1754.

22. Tandina F, Almeras L, Koné AK, Doumbo OK, Raoult D, Parola P, 2016. Use of MALDI-TOF MS and culturomics to identify mosquitoes and their midgut microbiota. Parasit Vectors 9: 945.

23. Raharimalala FN, Andrianinarivomanana TM, Rakotondrasoa A, Collard JM, Boyer S, 2017. Usefulness and accuracy of MALDITOF mass spectrometry as a supplementary tool to identify mosquito vector species and to invest in development of international database. Med Vet Entomol 31: 289-298.
24. Mewara A, Sharma M, Kaura T, Zaman K, Yadav R, Sehgal R, 2018. Rapid identification of medically important mosquitoes by matrix-assisted laser desorption/ionization time-of-flight mass spectrometry. Parasit Vectors 11: 281.

25. Yssouf A, Socolovschi C, Leulmi H, Kernif T, Bitam I, Audoly G, Almeras L, Raoult D, Parola P, 2014. Identification of flea species using MALDI-TOF/MS. Comp Immunol Microbiol Infect Dis 37: 153-157.

26. Karger A, Kampen H, Bettin B, Dautel H, Ziller M, Hoffmann B, Süss J, Klaus C, 2012. Species determination and characterization of developmental stages of ticks by whole-animal matrix-assisted laser desorption/ionization mass spectrometry. Ticks Tick Borne Dis 3: 78-89.

27. Yssouf A, Flaudrops C, Drali R, Kernif T, Socolovschi C, Berenger JM, Raoult D, Parola P, 2013. Matrix-assisted laser desorption ionization-time of flight mass spectrometry for rapid identification of tick vectors. J Clin Microbiol 51: 522-528.

28. Diarra $A Z$, Almeras $L$, Laroche $M$, Berenger J-M, Koné AK, Bocoum Z, Dabo A, Doumbo O, Raoult D, Parola P, 2017. Molecular and MALDI-TOF identification of ticks and tickassociated bacteria in Mali. PLoS Negl Trop Dis 11: e0005762.

29. Mathis $A$ et al., 2015. Identification of Phlebotomine sand flies using one MALDI-TOF MS reference database and two mass spectrometer systems. Parasit Vectors 8: 266.

30. Halada P, Hlavackova K, Risueño J, Berriatua E, Volf $P$, Dvorak V, 2018. Effect of trapping method on species identification of phlebotomine sandflies by MALDI-TOF MS protein profiling. Med Vet Entomol 32: 388-392.

31. Hoppenheit A, Murugaiyan J, Bauer B, Steuber S, Clausen PH, Roesler U, 2013. Identification of Tsetse (Glossina spp.) using matrix-assisted laser desorption/ionisation time of flight mass spectrometry. PLoS Negl Trop Dis. 7: e2305.

32. Kaufmann C, Schaffner F, Ziegler D, Pflüger V, Mathis A, 2012. Identification of field-caught Culicoides biting midges using matrix-assisted laser desorption/ionization time of flight mass spectrometry. Parasitology 139: 248-258.

33. Sambou M et al., 2015. Comparison of matrix-assisted laser desorption ionization-time of flight mass spectrometry and molecular biology techniques for identification of Culicoides (Diptera: ceratopogonidae) biting midges in Senegal. J Clin Microbiol 53: 410-418.

34. Lafri I, El Hamzaoui B, Bitam I, Leulmi H, Lalout R, Mediannikov O, Chergui M, Karakellah M, Raoult D, Parola P, 2017. Detection of relapsing fever Borrelia spp., Bartonella spp. and Anaplasmataceae bacteria in argasid ticks in Algeria. PLoS Negl Trop Dis 11: e0006064.

35. Laroche M, Bérenger JM, Gazelle G, Blanchet D, Raoult D, Parola $P, 2018$. MALDI-TOF MS protein profiling for the rapid identification of Chagas disease triatomine vectors and application to the triatomine fauna of French Guiana. Parasitology 145: 665-675.

36. Plichart C, Legrand AM, 2005. Detection and characterization of Wolbachia infections in Wuchereria bancrofti (Spirurida: Onchocercidae) var. pacifica and Aedes (Stegomyia) polynesiensis (Diptera: Culicidae). Am J Trop Med Hyg 73: 354-358.

37. Grjébine A, 1966. Insectes Diptères Culicidae Anophelinae. Paris, France: ORSTOM. Available at: http://www.documentation.ird. fr/hor/fdi:11315. Accessed August 9, 2019.

38. Niare S, Berenger JM, Dieme C, Doumbo O, Raoult D, Parola P, Almeras L, 2016. Identification of blood meal sources in the main African malaria mosquito vector by MALDI-TOF MS. Malar J 15: 87.

39. Nebbak A, Willcox AC, Bitam I, Raoult D, Parola P, Almeras L, 2016. Standardization of sample homogenization for mosquito identification using an innovative proteomic tool based on protein profiling. Proteomics 16: 3148-3160.

40. Laroche M, Almeras L, Pecchi E, Bechah Y, Raoult D, Viola A, Parola P, 2017. MALDI-TOF MS as an innovative tool for detection of Plasmodium parasites in Anopheles mosquitoes. Malar J 16: 5.

41. Diarra AZ, Laroche M, Berger F, Parola P, 2019. Use of MALDITOF MS for the identification of Chad mosquitoes and the origin of their blood meal. Am J Trop Med Hyg 100: 47-53.

42. Benkacimi L, Gazelle G, El Hamzaoui B, Bérenger JM, Parola P, Laroche M, 2020. MALDI-TOF MS identification of Cimex 
lectularius and Cimex hemipterus bedbugs. Infect Genet Evol 85: 104536.

43. Smith JL, Fonseca DM, 2004. Rapid assays for identification of members of the Culex (Culex) pipiens complex, their hybrids, and other sibling species (Diptera: Culicidae). Am J Trop Med Hyg 70: 339-345.

44. Tandina F, Niaré S, Laroche M, Koné AK, Diarra AZ, Ongoiba A, Berenger JM, Doumbo OK, Raoult D, Parola P, 2018. Using MALDI-TOF MS to identify mosquitoes collected in Mali and their blood meals. Parasitology 145: 1170-1182.

45. El Hamzaoui B, Laroche M, Parola P, 2019. Detection of Bartonella spp. in Cimex lectularius by MALDI-TOF MS. Comp Immunol Microbiol Infect Dis 64: 130-137.

46. Lawrence AL, Batovska J, Webb CE, Lynch SE, Blacket MJ, Ślapeta J, Parola P, Laroche M, 2019. Accurate identification of Australian mosquitoes using protein profiling. Parasitology 146: 462-471.

47. Normand AC et al., 2017. Validation of a new web application for identification of fungi by use of matrix-assisted laser desorption ionization-time of flight mass spectrometry. J Clin Microbiol 55: 2661-2670.

48. Centers for Disease Control and Prevention, 2018. MicrobNet. Available at: https://www.cdc.gov/microbenet/index.html. Accessed October 15, 2020.

49. Dvorak V, Halada P, Hlavackova K, Dokianakis E, Antoniou M, Volf $P, 2014$. Identification of phlebotomine sand flies (Diptera: Psychodidae) by matrix-assisted laser desorption/ionization time of flight mass spectrometry. Parasit Vectors 7: 21.

50. Carnevale P, Robert V, 2009. Les anophèles: Biologie, transmission du Plasmodium et lutte antivectorielle. Paris, France: IRD Éditions.

51. Mouchet J, Carnevale P, Coosemans M, Jul J, 2004. Biodiversité du paludisme dans le monde. Paris, France: John libbey eurotext, 1.

52. Raikhel AS, Kokoza VA, Zhu J, Martin D, Wang SF, Li C, Sun G, Ahmed A, Dittmer N, Attardo G, 2002. Molecular biology of mosquito vitellogenesis: from basic studies to genetic engineering of antipathogen immunity. Insect Biochem Mol Biol 32: $1275-1286$. 